\title{
Efecto del tabaco sobre la evolución de los tumores de vejiga
}

\author{
García Mediero JM, Angulo Cuesta J, Luján Galán M, Rodríguez García N, Berenguer Sánchez A.
}

Servicio de Urología. Hospital Universitario de Getafe, Madrid.

Actas Urol Esp. 2007;31(4):349-354

\section{RESUMEN}

EFECTO DEL TABACO SOBRE LA EVOLUCIÓN DE LOS TUMORES DE VEJIGA

Objetivos: Evaluar las características de las neoplasias vesicales en pacientes fumadores en el momento del diagnóstico y estudiar las diferencias en recurrencia y progresión de los pacientes fumadores y los no fumadores. Pacientes y Métodos: Se revisaron 786 historias clínicas de pacientes con tumores vesicales de nuestra base de datos de una forma retrospectiva desde 1991 al 2004 y se analizaron las características tumorales así como el evento recurrencia y progresión de los pacientes fumadores frente a los no fumadores. Resultados: 377 (48\%) pacientes eran fumadores al momento del diagnóstico. No encontramos diferencias significativas en cuanto a edad ni a categoría T tumoral entre ambos grupos (t student y Chi cuadrado). Sin embargo, si encontramos diferencias estadísticamente significativas en cuanto a grado tumoral, tamaño tumoral ( mayor o menor de $2 \mathrm{~cm}$.) y multiplicidad (Chi cuadrado, $\mathrm{p}=0,046, \mathrm{p}=0,001 \mathrm{y} \mathrm{p}=0,012$ respectivamente). No encontramos diferencias significativas para la recidiva.(Log Rank, Breslow y Tarone-Ware N.S.). En cuanto a progresión si se aprecia una diferencia estadísticamente significativa entre fumadores y no fumadores en el estudio univariante $(\log R a n k \mathrm{p}=0,03$, Breslow $\mathrm{p}=0,05$ y Tarone-Ware $\mathrm{p}=0,03$ ) aunque no soporta el estudio multivariante. Conclusiones: No puede reconocerse el hábito tabáquico como un factor independiente de recurrencia o progresión en los tumores superficiales. Sin embargo, los fumadores debutan más frecuentemente con tumores de alto grado, de una manera múltiple y de un tamaño igual o superior a $2 \mathrm{~cm}$. de una manera significativa frente a los no fumadores.

Palabras clave: Tabaquismo. Cáncer vesical.

\section{ABSTRACT \\ SMOKING HABIT AND SUPERFICIAL BLADDER TUMOURS NATURAL HISTORY}

Objectives: To disclose the features of bladder cancer in current smokers at diagnosis and to study the differences in recurrence and progression between smokers and non-smokers. Patients \& Methods: 786 consecutive patients with bladder cancer from 1991 to 2004 were studied in a retrospective manner and characteristics of smokers tumours were compared to non smokers. Results: 377 (48\%) patients were smokers at diagnosis. There were no differences in terms of age and T stage between smokers and non smokers (t student and Chi square). We found statistical differences in tumours grade, size and multiplicity ( $C h i$ square, $\mathrm{p}=0.046, \mathrm{p}=0.001$ and $\mathrm{p}=0.12$ respectly). No differences were found between smokers and non smokers according to recurrence. (Log Rank, Breslow y Tarone-Ware N.S.). We did find differences according to progression (Log Rank $\mathrm{p}=0.03$, Breslow $\mathrm{p}=0.05$ y Tarone-Ware $\mathrm{p}=0.03$ ) although it did not support multivariate study. Conclusions: Currents smokers present bigger and higher grade tumors and, more frequently in a multiple fashion at diagnosis than non-smokers.

Keywords: Smoking habit. Bladder cancer. 
A unque el hábito tabáquico se conoce como factor de riesgo para el desarrollo de tumores vesicales, son escasos los trabajos que estudian si existe alguna variación en la historia natural del cáncer vesical en fumadores; tanto a nivel de señalar características distintivas en los tumores que desarrolla la población fumadora como en el riesgo de recidiva y/o progresión de la enfermedad. Este estudio analiza el papel del tabaquismo activo sobre la evolución del cáncer vesical.

El consumo de cigarrillos supone la causa aislada más importante para desarrollar un carcinoma de vejiga (CAV) y los fumadores presentan un riesgo entre dos y cuatro veces más elevado de sufrir un CAV que la población general ${ }^{1}$.

El humo de los cigarrillos presenta, entre otros, aldehídos insaturados y aminas aromáticas que parecen ser los responsables de este riesgo. El aumento del riesgo de los sujetos fumadores continúa durante varios años, entre 12 y 15 , después de abandonar el hábito ${ }^{1}$. Entre los diferentes tipos de tabaco aquel secado al aire en su producción (tabaco negro) presenta mayor riesgo de producir CAV que aquel secado en estufas (tabaco rubio) $^{2}$.

Aunque Thompson et al. ${ }^{3}$ demostraran una correlación entre el tabaco y el grado histológico, el estadio, número y tamaño de los tumores vesicales otros estudios no han podido reproducir estos hallazgos ${ }^{4}$. Entre estos últimos, Raitamen si demostró una disminución de la supervivencia a los 10 años de los fumadores con CAV frente a los no fumadores ${ }^{4}$.

\section{MATERIAL Y MÉTODOS}

Se revisaron 786 historias clínicas de pacientes con tumores vesicales de nuestra base de datos de una forma retrospectiva desde 1991 al 2004 y se analizaron tanto las características epidemiológicas (edad, sexo) como las tumorales (número, tamaño - empleando como corte $2 \mathrm{~cm}-$, grado tumoral y categoría $\mathrm{T}$ ) de los pacientes fumadores frente a los no fumadores al diagnóstico. Para el estudio estadístico se emplearon el test de ANOVA para establecer si existen diferencias significativas respecto a la edad del diagnóstico y Chi-cuadrado respecto al sexo, categoría $\mathrm{T}$, número de implantes y tamaño tumoral. Para identificar factores predisponentes a la recidiva y progresión, se exploraron las relaciones entre las características de los pacientes y sus tumores, y la recidiva y progresión. Como comprobación multivariante, se llevó a cabo un análisis por medio de un modelo de regresión de COX utilizando una estrategia de eliminación hacia atrás. En cada paso, se eliminó la variable con el valor de $\mathrm{P}$ más elevado. El modelo máximo incluyó la edad, el sexo, la categoría $\mathrm{T}$, el grado tumoral, el tamaño, aspecto y el número de tumores y el hábito de fumar. Las proyecciones de supervivencia libre de enfermedad. (SLE) y supervivencia libre de progresión, se analizaron mediante curvas de Kaplan-Meier. Para todas las comparaciones estadísticas se utilizó un nivel de confianza del 95\%. Para el análisis se utilizó un programa bioestadístico comercial: SPSS 11,5.

\section{RESULTADOS}

Un total de 377 (48\%) pacientes eran fumadores en el momento del diagnóstico, con una mediana de edad al diagnóstico de 65 años (16-94). Veinte de un total de 70 mujeres fueron fumadoras $(28,6 \%)$ frente a un $34 \%$ de varones fumadores (Chi cuadrado NS). No encontramos tampoco diferencias significativas en cuanto a la edad al diagnóstico entre los dos grupos de pacientes (ANOVA N.S.). Tampoco encontramos diferencias estadísticamente significativas entre ambas poblaciones de pacientes en cuanto a la categoría $\mathrm{T}$ al diagnóstico (Chi cuadrado NS) (Fig. 1).

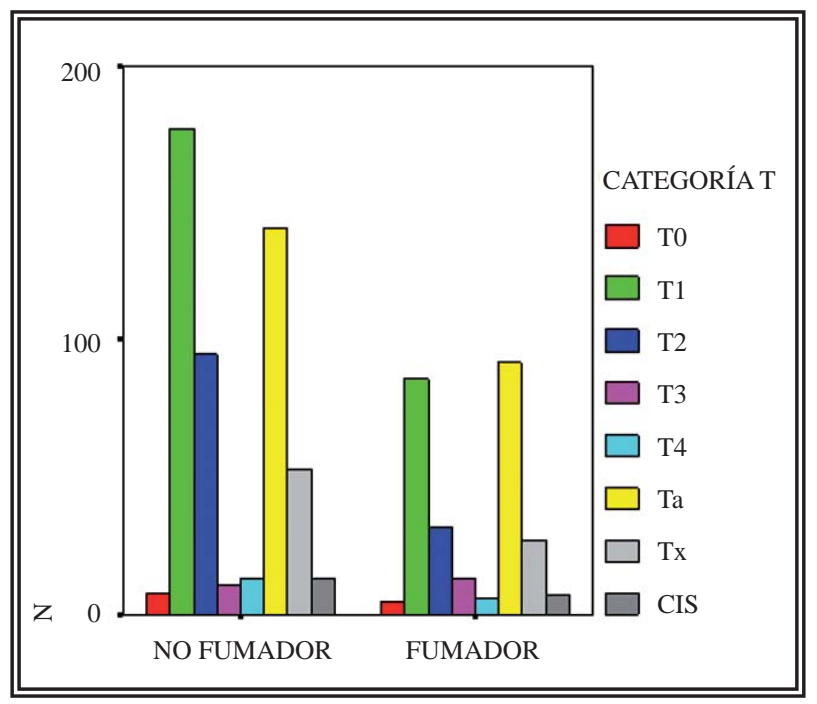

FIGURA 1. Categoría $T$ al diagnóstico en las dos poblaciones de pacientes al diagnóstico: fumadores y no fumadores. No encontramos diferencias estadisticamente significativas entre ambas poblaciones (Chi cuadrado NS). 
Los pacientes fumadores al diagnóstico presentaron significativamente más tumores de alto grado G3 que los no fumadores (Chi cuadrado $\mathrm{p}=0,046$ ) (Fig. 2). En cuanto al tamaño tumoral, también encontramos diferencias significativas, siendo mayores los tumores en los pacientes fumadores que en los no fumadores (Chi cuadrado $\mathrm{p}=0,001$ ) (Fig. 3). Agrupamos el número de implantes tumorales en la resección de vejiga como ÚNICOS o MÚLTIPLES y los pacientes fumadores presentaron más de un tumor al diagnóstico frente a los no fumadores (Chi cuadrado $\mathrm{p}=0,012$ ) (Tabla 1). 22 pacientes fumadores presentaron CIS asociado a la neoformación vesical frente a 12 no fumadores con esta neoplasia asociada (Chi cuadrado $\mathrm{p}=0,001$ ).

Con una mediana de seguimiento de 16,4 meses, la supervivencia libre de enfermedad de los pacientes fumadores al diagnóstico fue del 7\% mientras que la de los no fumadores fue del $19 \%$ (mediana de seguimiento de 20 meses). Es decir, la mitad de los pacientes a estudio recidivaron a los 16,4 y 20 meses respectivamente, pero no encontramos diferencias significativas entre ambas curvas de supervivencias (Log Rank, Breslow y Tarone-Ware N.S.) (Fig. 4).

En cuanto a la progresión a tumores músculo infiltrantes de los tumores superficiales, si se aprecia una diferencia estadísticamente significativa entre fumadores y no fumadores como se aprecia en la Figura 5 (Log Rank $p=0,03$, Breslow $\mathrm{p}=0,05$ y Tarone-Ware $\mathrm{p}=0,03$ ). La función del riesgo de progresión nos indica que tal riesgo es muy patente en los 200 primeros meses pero que continúa elevándose continuamente, siendo el riesgo mayor en los fumadores que en los no fumadores (Fig. 6).

Sin embargo, al realizar un análisis multivariante (Regresión logística de COX) en donde el modelo máximo incluyó la edad, el sexo, la categoría $\mathrm{T}$ (en los tumores superficiales), el grado tumoral, el tamaño, aspecto y el número de tumores y el hábito de fumar, los únicos factores predictivos para el evento recurrencia fueron el grado tumoral $(\mathrm{p}=0,005)$ y la multi-

Tabla 1 cuadrado $\mathrm{p}=0,012$ ).

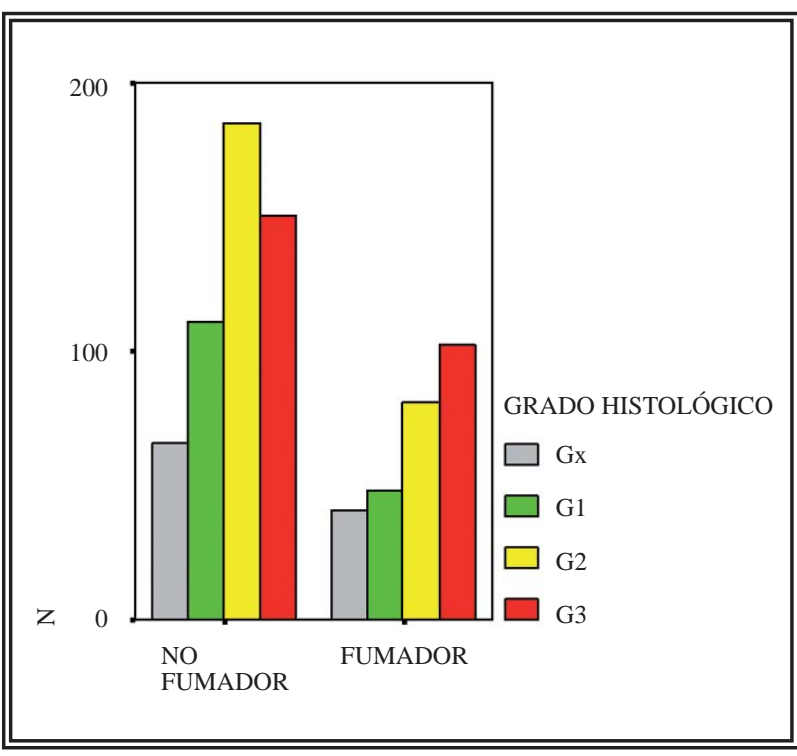

FIGURA 2 Los pacientes fumadores al diagnóstico pre sentaron significativamente más tumores de alto grado G3 que los no fumadores (Chi cuadrado $p=0,046$ ).

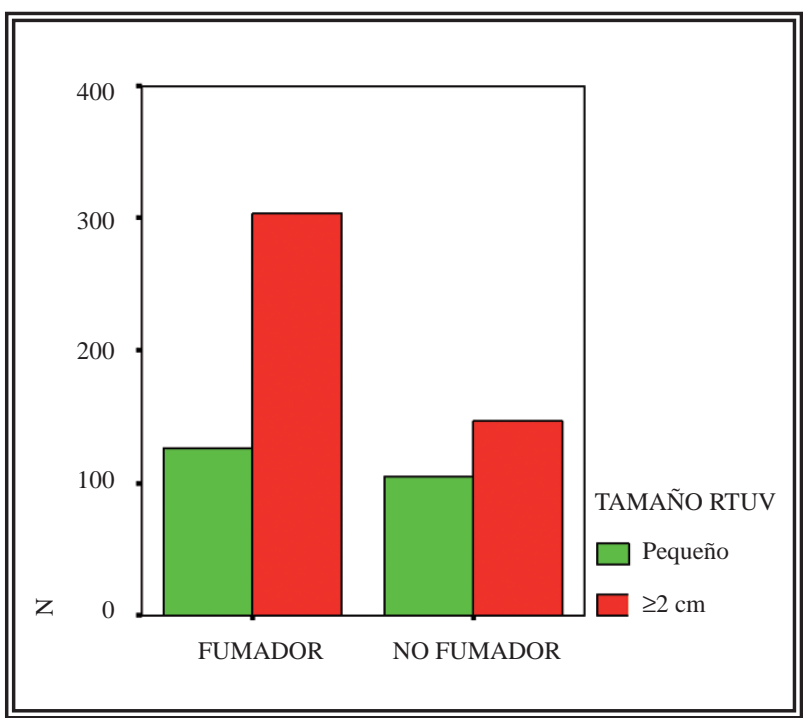

FIGURA 3. Los tumores en los pacientes fumadores son significativamente mayores que en los no fumadores (Chi cuadrado $p=0,001$ ).

\begin{tabular}{llccccc}
\hline & & & \multicolumn{2}{c}{ Número tumores } & & \multirow{2}{*}{ Total } \\
& & No tumor & Únicos & Múltiples & Perdidos & \\
\hline \multirow{2}{*}{ Tabaco } & Fumador & 1 & 310 & 128 & 74 & 513 \\
& No fumador & 3 & 164 & 84 & 22 & 273 \\
\multirow{2}{*}{ Total } & & 4 & 474 & 212 & 96 & 786 \\
\hline
\end{tabular}

Tabla de contingencia de fumadores/no fumadores frente a tumores únicos/ múltiples. Los fumadores presentaron de forma significativa más de un tumor frente a los no fumadores( Chi 


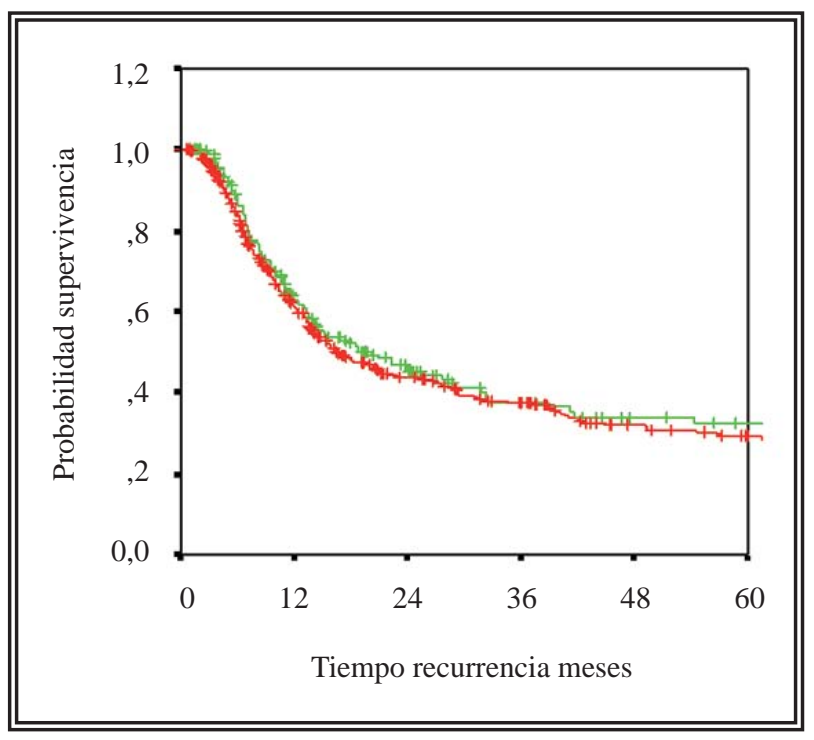

FIGURA 4. Proyección de supervivencia libre de enfermedad (recidiva) de los pacientes fumadores frente a los no fumadores. No hay diferencias estadisticamente significativas entre ambas curvas (Log Rank, Breslow y TaroneWare N.S.).

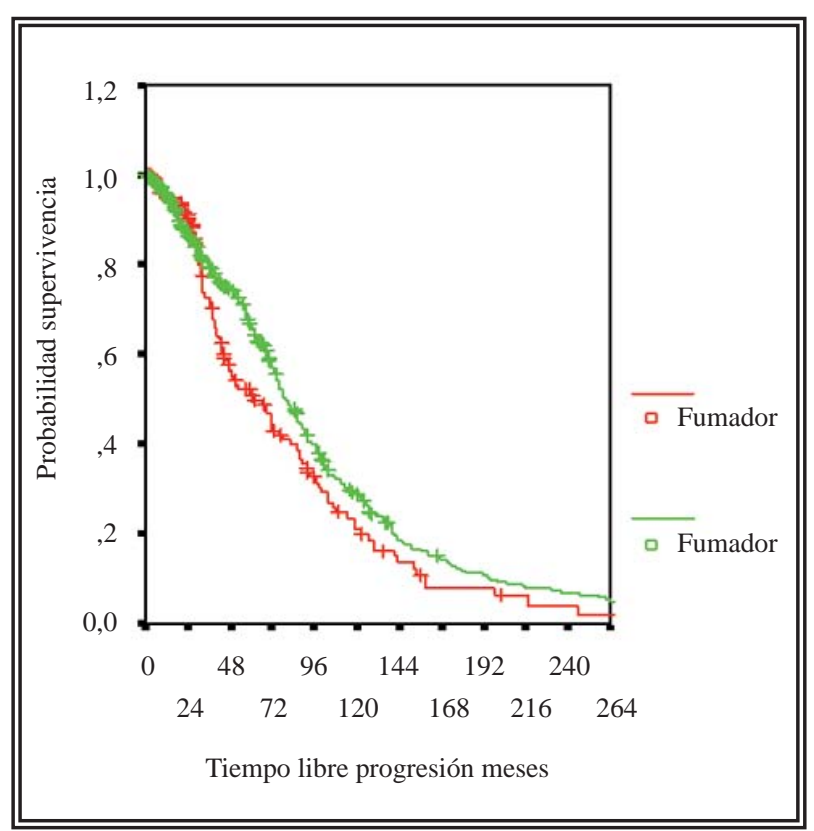

FIGURA 5. Proyección de supervivencia libre de progresión. Se aprecia una diferencia estadisticamente significativa entre fumadores $y$ no fumadores (Log Rank $p=0,03$, Breslow $p=0,05$ y Tarone-Ware $p=0,03$ ).

plicidad de los mismos ( $\mathrm{p}=0,000)$.El mismo análisis para predecir progresión tumoral (excluyendo T2-T4) no nos permite identificar una única covariable significativamente estadística para predecir el evento Progresión.

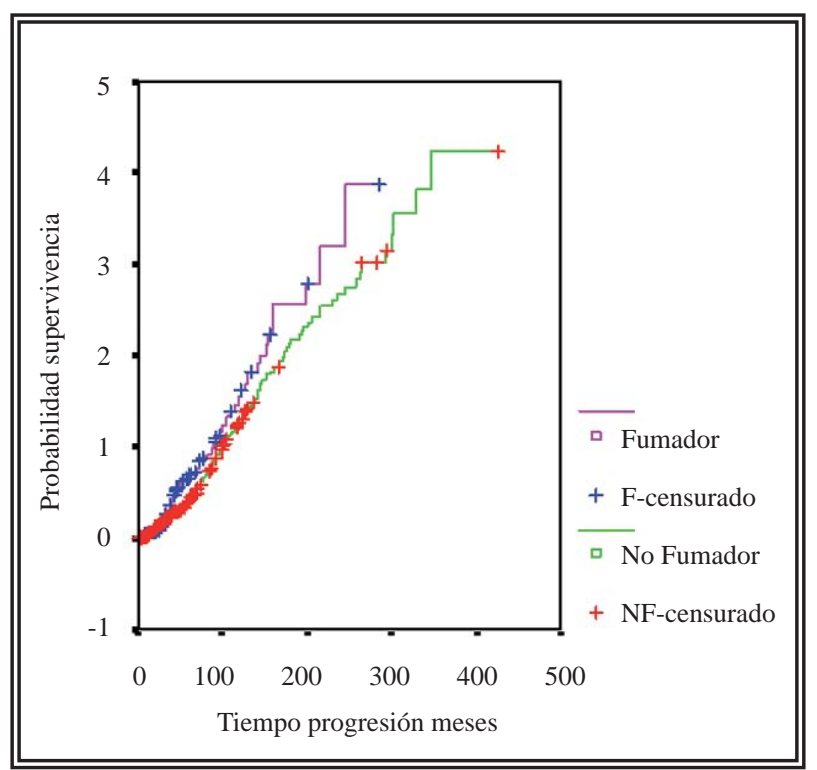

FIGURA 6. Curva de función del riesgo de progresión. El riesgo continúa elevándose con el paso del tiempo, de forma más importante en los fumadores que en los no fumadores.

\section{DISCUSIŌN}

El consumo de cigarrillos supone la causa aislada más importante para desarrollar un carcinoma vesical y los fumadores presentan un riesgo entre dos y cuatro veces más elevado de sufrir esta neoplasia que la población general ${ }^{2}$. El humo de los cigarrillos presenta, entre otros, aldehídos insaturados y aminas aromáticas que parecen ser los responsables de este riesgo. El aumento del riesgo de los sujetos fumadores continúa durante varios años, entre 12 y 15, después de abandonar el hábito ${ }^{2}$. En nuestra Comunidad Autónoma de Madrid, existen, según los datos del INE ${ }^{5} 122730$ personas que fuman al menos 3 ó 4 veces a la semana. No tenemos recogido en nuestra serie el número de cigarrillos/ día en los pacientes fumadores. Por esta razón no podemos realizar un análisis de tendencia lineal para estudiar la relación entre la cantidad de tabaco consumido y el aumento del riesgo de padecer neoplasia vesical, recidiva tumoral y progresión. Así las cosas, nos hemos centrado en la variable "hábito de fumar", tomándola como dicotómica y de esta manera, poder estudiar el efecto global del tabaco.

En nuestra población de pacientes con neoplasias vesicales encontramos un gran porcentaje de los mismos que son fumadores, quizás sea rele- 
vante el hecho que es un área fundamentalmente industrial del Sur de Madrid. También es interesante resaltar que un tercio de las mujeres con neoplasia vesical son fumadoras al diagnóstico. Este factor ya se estudió en los Estados Unidos al apreciar un aumento de incidencia del cáncer de pulmón en mujeres al incorporarse estas al mundo laboral a finales de los años sesenta ${ }^{6}$.

Encontramos diferencias estadísticamente significativas entre los pacientes que fuman en cuanto al grado tumoral (son de alto grado), la multiplicidad (son múltiples en los fumadores ) y el tamaño tumoral (mayores en los fumadores), aunque solo las dos primeras covariables soportan el análisis multivariante en cuanto a recidiva tumoral. Y según los datos de nuestra serie, el hábito de fumar, aunque significativamente aumenta la probabilidad de progresión tumoral, no se confirma este hecho en presencia del resto de las covariables al realizar el análisis de regresión multivariante.

Aunque Thompson et $\mathrm{al}^{3}$ demostraran una correlación entre el tabaco y el grado histológico, el estadio, número y tamaño de los tumores vesicales otros estudios no consiguieron reproducir$1 \mathrm{o}^{3}$. Raitamen si demostró una disminución de la supervivencia a los 10 años de los fumadores con CAV frente a los no fumadores ${ }^{4}$.

Las diferencias encontradas en los diferentes trabajos pueden ser explicadas por los diferentes tipos de degradación de las aminas aromáticas.

Las dos vías principales de degradación de estas son la vía de la $\mathrm{N}$-acetilación y la de la $\mathrm{N}$ hidroxilación. Los productos de la primera vía no son carcinógenos mientras que los productos de la segunda si lo son. La enzima clave en la primera vía es la $\mathrm{N}$-acetiltransferasa 2 (NAT2) y existen dos genotipos para esta enzima, que resultan en dos tipos de sujetos, los "acetiladores lentos" y los "rápidos"? . Como los compuestos acetilados son menos carcinogénicos, los sujetos acetiladores rápidos serán menos sensibles a la exposición al humo del tabaco ${ }^{8}$.

En 1998, Taylor et al. demostraron que los varones con antecedentes de 30 años de tabaquismo que además eran homocigotos para el gen que codifica la N-acetiltransferasa 1 (NAT1) presentaban un riesgo incluso mayor (aumento de 8,5 veces) que los homocigotos para la NAT2 ${ }^{9}$. El genotipo de estas NAT podría explicar las diferencias en incidencia y prevalencia del CAV presentes entre las diferentes razas ${ }^{10}$. Otras enzimas involucradas en la patogénesis tumoral del cáncer de vejiga por el tabaco son el citocromo P450 1A2 (CYP 1A2), que demetila aminas aromáti$\operatorname{cas}^{11} \mathrm{y}$ la glutation-S-transferasa $\mathrm{M} 1^{12}$.

El estudio de las vías de degradación de los carcinógenos del tabaco y el tipo de daño que realizan a nivel molecular sobre el DNA y sobra áreas específicas del genoma relacionadas con el cáncer de vejiga (ej. P53) arrojará luz sobre su mecanismo de acción y sobre posibles estrategias terapéuticas ${ }^{13}$.

\section{CONCLUSIONES}

No puede reconocerse el hábito tabáquico como un factor independiente de recurrencia o progresión en los tumores superficiales; ahora bien, existe cierta evidencia para considerar que los pacientes que se reconocen fumadores activos en el momento del diagnóstico tienen mayor riesgo de presentar lesiones de mayor grado, tamaño $\mathrm{y}$ frecuencia de CIS asociado, e incluso tumores con mayor riesgo de progresión. Los esfuerzos de salud pública dirigidos al abandono de este hábito pueden resultar beneficiosos para los pacientes con cáncer vesical.

\section{REFERENCIAS}

1. Wynder EL, Goldsmith R. The epidemiology of bladder cancer: a second look. Cancer. 1977;40(3):1246-1268.

2. R. Lee y M. Droller. : "Evolución natural del cáncer de vejiga”, Clínicas Urológicas de Norteamérica, Vol 1 (2000), 1-13, Ed McGraw-Hill Interamericana.

3. Thompson IM, Peek M, Rodriguez F. The impact of cigarette smoking on stage, grade and number of recurrences of transitional cell carcinoma of the bladder. J Urol. 1987;137(3): 401-403.

4. Raitanen MP, Nieminen P Tammela TL. Impact of tumour grade, stage, number and size, and smoking and sex, on survival in patients with transitional cell carcinoma of the bladder. Br J Urol. 1995;76(4):470-474.

5. www.ine.es.

6. Greenlee RT, Murray T, Bolden S, Wingo PA. Cancer statistics, 2000. CA Cancer J Clin. 2000;50(1):7-33

7. Cartwright RA, Glashan RW, Rogers HJ, Ahmad RA, Barham-Hall D, Higgins E, et al. Role of N-acetyltransferase phenotypes in bladder carcinogenesis: A pharmacogenetic epidemiologic approach to bladder cancer. Lancet. 1982;16; 2(8303):842-845. 
8. Risch A, Wallace DM, Bathers S, Sim E. Slow N-acetylation genotype is a susceptibility factor in occupational and smoking related bladder cancer. Hum Mol Genet. 1995; 4(2): 231-236.

9. Taylor JA, Umbach DM, Stephens E, Castranio T, Paulson $\mathrm{D}$, Robertson $\mathrm{C}$, et al. The role of N-acetylation polymorphisms in smoking associated bladder cancer: Evidence of gene-gene-exposure three-way interaction. Cancer Res. 1998;58(16):3603-3610.

10. Yu MC, Skipper PL, Taghizadeh K, Tannenbaum SR, Chan $\mathrm{KK}$, Henderson BE et al. Acetylator phenotype, Aminobiphenyl-hemoglobin adduct levels, and bladder cancer risk in white, black, and Asian men in Los Angeles, California. J Natl Cancer Inst. 1994;86(9):712-716.

11. Horn EP, Tucker MA, Lambert G, Silverman D, Zametkin D, Sinha R et al. A study of gender-based cytochrome P450 1A2 variability: A possible mechanism for the male excess of bladder cancer. Cancer Epidemiol Biomarkers Prev. 1995;4(5):529-533.
12. Bell DA, Taylor JA, Paulson DF, Robertson CN, Mohler JL Lucier GW. Genetic risk and carcinogen exposure: a common inherited defect of the carcinogen-metabolism gene glutathione S-transferase M1 (GSTM1) that increases susceptibility to bladder cancer. J Natl Cancer Inst. 1993;85 (14):1159-1164.

13. Jones PA, Buckley JD, Henderson BE, Ross RK Pike MC. From gene to carcinogen: A rapidly evolving field in molecular epidemiology. Cancer Res. 1991;51(13):3617-3620.

Correspondencia autor: Dr. J.M. García Mediero Servicio de Urología. Hospital Universitario de Getafe Ctra. de Toledo, km. 12,500. 28905 Getafe. Madrid. Tel.: 916839360

E-mail autor: garciamediero@hotmail.com Información artículo: Original - Cáncer de vejiga

Trabajo recibido: octubre 2006

Trabajo aceptado: enero 2007 